OPEN ACCESS

Edited by:

Thomas Dandekar,

University of Würzburg, Germany

Reviewed by:

Laurence S. Dawkins-Hall,

University of Leicester,

United Kingdom

Paras Jain,

Albert Einstein College of Medicine,

United States

*Correspondence:

Michael G. Sehorn

msehorn@clemson.edu

Specialty section:

This article was submitted to

Infectious Diseases,

a section of the journal

Frontiers in Microbiology

Received: 10 June 2017

Accepted: 24 August 2017

Published: 07 September 2017

Citation:

Kelso AA, Waldvogel SM

Luthman AJ and Sehorn MG (2017)

Homologous Recombination

in Protozoan Parasites

and Recombinase Inhibitors.

Front. Microbiol. 8:1716.

doi: 10.3389/fmicb.2017.01716

\section{Homologous Recombination in Protozoan Parasites and Recombinase Inhibitors}

\author{
Andrew A. Kelso ${ }^{1,2}$, Sarah M. Waldvogel' ${ }^{1}$, Adam J. Luthman ${ }^{1}$ and \\ Michael G. Sehorn ${ }^{1,2,3,4 *}$
}

${ }^{1}$ Department of Genetics and Biochemistry, Clemson University, Clemson, SC, United States, ${ }^{2}$ Eukaryotic Pathogens Innovation Center, Clemson University, Clemson, SC, United States, ${ }^{3}$ Center for Optical Materials Science and Engineering Technologies, Clemson University, Clemson, SC, United States, ${ }^{4}$ Clemson University School of Health Research, Clemson University, Clemson, SC, United States

Homologous recombination (HR) is a DNA double-strand break (DSB) repair pathway that utilizes a homologous template to fully repair the damaged DNA. HR is critical to maintain genome stability and to ensure genetic diversity during meiosis. A specialized class of enzymes known as recombinases facilitate the exchange of genetic information between sister chromatids or homologous chromosomes with the help of numerous protein accessory factors. The majority of the HR machinery is highly conserved among eukaryotes. In many protozoan parasites, HR is an essential DSB repair pathway that allows these organisms to adapt to environmental conditions and evade host immune systems through genetic recombination. Therefore, small molecule inhibitors, capable of disrupting HR in protozoan parasites, represent potential therapeutic options. A number of small molecule inhibitors were identified that disrupt the activities of the human recombinase RAD51. Recent studies have examined the effect of two of these molecules on the Entamoeba recombinases. Here, we discuss the current understandings of HR in the protozoan parasites Trypanosoma, Leishmania, Plasmodium, and Entamoeba, and we review the small molecule inhibitors known to disrupt human RAD51 activity.

Keywords: homologous recombination, DNA repair, double-strand break repair, protozoan parasites, recombination inhibitors, RAD51, DMC1

\section{INTRODUCTION}

DNA double-stranded breaks (DSBs) can occur due to exogenous or endogenous events. Exogenous sources of DSBs include reactive oxygen species generated from exposure to ionizing radiation and radiomimetic chemicals (Mehta and Haber, 2014), while endogenous sources of DSBs include erroneous DNA replication that can lead to the collapse of replication forks. Additionally, DSBs can be intentionally introduced into the genome through programmed events such as in meiosis, where the production of crossover products is essential. Whether they are produced intentionally or as a result of genotoxic events, unrepaired DSBs threaten the genome stability of an organism. Defects in DSB repair pathways have been associated with sterility, cancer, and chromosomal rearrangements (San Filippo et al., 2008; Moynahan and Jasin, 2010; Hunter, 2015). 
Four major pathways function in DSB repair: nonhomologous end joining (NHEJ), single-strand annealing (SSA), alternative end joining (ALT-EJ), and homologous recombination (HR) (Figure 1). NHEJ (also known as classicalNHEJ or canonical-NHEJ) is the major DSB repair pathway, and is mediated by many factors, which include Ku70, Ku80, XRCC4, and DNA ligase IV (Lieber, 2010). In this pathway, the ends of the DSB are protected to prevent nucleolytic end resection (Mimori and Hardin, 1986; Paillard and Strauss, 1991), followed by ligation to mend the DSB (Figure 1A). NHEJ is active during the entire cell cycle, and is the pathway responsible for $\mathrm{V}(\mathrm{D}) \mathrm{J}$ recombination in antibody variation (Soulas-Sprauel et al., 2007; Malu et al., 2012). NHEJ can result in full restoration of the DSB. However, if there is any enzymatic processing or degradation at the ends of the DSB, there will be a loss of genetic information once repaired (Betermier et al., 2014); thus, NHEJ is often associated with chromosomal alterations at the break site (Ghezraoui et al., 2014). Repair by SSA is initiated when a DSB occurs at a locus with extensive homology in the sequences flanking the break site (Figure 1B). Once homology is located ( $\geq 100$ base pairs), the sequences are annealed by the DNA annealing factor RAD52 (Morales et al., 2015) and the non-homologous overlapping ends are endonucleolytically processed followed by DNA polymerase gap filling and ligation to restore the break (Bhargava et al., 2016). Similarly, ALT-EJ (also known as a microhomology mediated-EJ) DSB repair occurs through the recognition and annealing of short sequences called microhomology ( $\leq 10$ base pairs) within the sequences flanking the DSB (Wang and $\mathrm{Xu}$, 2017) (Figure 1C). The two main protein factors involved in mediating ALT-EJ are poly-ADP-ribose polymerase (PARP) and DNA polymerase theta (POLQ) (Bhargava et al., 2016). SSA and ALT-EJ are both active early in the S and G2 phases of the cell cycle. Both pathways result in the loss of DNA sequence between the annealed substrates (SSA results in large deletion events and ALT-EJ results in small deletions). As a result, both SSA and ALT-EJ are considered to be mutagenic DSB repair pathways (Bhargava et al., 2016). In contrast, $\mathrm{HR}$ relies on a homologous template to restore the damaged DNA in its entirety (Figure 1D). HR is active during the $S$ and G2 phases of the cell cycle due to the presence of sister chromatids or homologous chromosomes (in meiosis) to act as the template. The HR pathway requires enzymatic resection of the ends of the DSB to produce $3^{\prime}$ single-stranded DNA (ssDNA) overhangs. These overhangs serve as the nucleation site for a recombinase, the central enzyme of HR. There are two conserved recombinases among eukaryotes, Rad51 (radiationsensitive 51) and Dmc1 (disrupted meiotic cDNA 1) (Moore, 1978; Bishop et al., 1992). Rad51 is the lead recombinase during mitosis, and Dmc1 serves as the major recombinase during meiosis, in which Rad51 plays a supportive role (Bishop, 2012). These different processes result in the requirement for different accessory proteins to modulate the functions of each recombinase. Nonetheless, the mechanisms of action for both recombinases are similar. The active ATP-bound form of the recombinase forms a right-handed presynaptic, nucleoprotein filament on the ssDNA overhang. The presynaptic filament then conducts a homology search to identify a homologous sequence that will serve as a template for repair. Rad51 preferentially utilizes the sister chromatid as a template in order to prevent loss of heterozygosity, whereas Dmcl prefers the homologous chromosome with the goal of generating crossover products. In either case, the presynaptic filament invades the duplex DNA and facilitates the base-pairing of the $3^{\prime}$ ssDNA with its complementary sequence. As a result, the homologous strand of the duplex DNA is displaced to form a displacement loop (D-loop) structure. The $3^{\prime}$ end of the invading strand primes DNA synthesis by a polymerase to replace DNA that was lost at the DSB site. As a result of strand invasion and DNA synthesis to repair the damaged DNA, two distinct DNA joint molecules can be formed. These DNA intermediates (the initial D-loop or Holliday junctions, which are associated with meiosis and form after D-loop extension, second end capture, ligation, and a second synthesis event) are resolved by one of two major pathways to ultimately yield non-crossover products or crossover products (Matos and West, 2014; Morrical, 2015).

The complex process of DSB repair by HR requires numerous accessory proteins to proceed with accuracy and efficiency (Table 1). RPA (replication protein A) is a heterotrimeric complex composed of three subunits (RPA1, RPA2, RPA3) that coat the ssDNA overhangs to protect them from nucleases and to prevent the formation of secondary structure (Binz et al., 2004). Although the DNA binding activity of RPA is necessary for $\mathrm{HR}$, this function also represents a barrier to the recombinase loading. Various recombination mediators help to overcome this inhibition by displacing RPA and loading the recombinase onto the ssDNA, including Saccharomyces cerevisiae Rad52 (radiationsensitive 52) and human BRCA2 (breast cancer susceptibility gene 2) (Sung, 1997; Jensen et al., 2010; Liu et al., 2010; Thorslund et al., 2010). Recently, DSS1 (deleted in split hand/split foot syndrome) was shown to aid BRCA2 in loading RAD51 onto RPA-coated ssDNA (Yang et al., 2002; Zhao et al., 2015). DSS1 functions as a DNA mimic that competes with authentic DNA to displace RPA, allowing BRCA2 to load RAD51 onto ssDNA (Zhao et al., 2014). Another factor, Hop2-Mnd1 (homologouspairing protein 2-meiotic nuclear division protein 1), is a meiosis-specific heterodimeric protein complex that promotes recombinase-meditated D-loop formation by stabilizing the presynaptic filament and bringing the duplex DNA into close proximity with the presynaptic filament for more efficient homologous DNA pairing (Chi et al., 2007; Pezza et al., 2007). Other accessory proteins, such as RAD54 (radiation-sensitive 54 ), promote the search for homology by the recombinase and help to dissociate the recombinase from the DNA after strand exchange has occurred (Petukhova et al., 1998; Mason et al., 2015). The high degree of conservation of the HR pathway across divergent eukaryotic species emphasizes its indispensable nature. The clinical relevance of $\mathrm{HR}$ in non-communicable human disease, such as cancer, makes it a potential therapeutic target. Furthermore, since HR plays a role in virulence of eukaryotic pathogens (see below), the components of this DNA repair system may also serve as a target for the development of new drugs to fight infectious disease. 


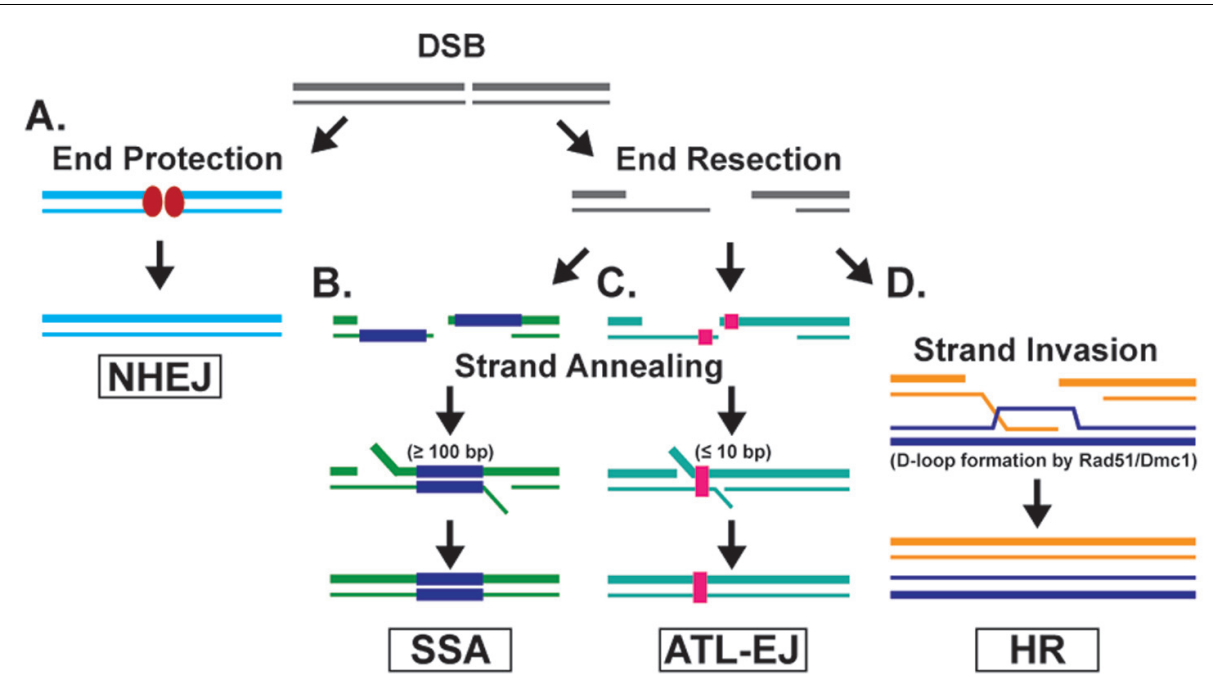

FIGURE 1 | Double-strand Break Repair Pathways. (A) The non-homologous end-joining (NHEJ) pathway is facilitated when the ends of the DSB are protected from resection and then ligated to mend the DSB. (B) Single-strand annealing (SSA) is a DSB repair pathway that anneals long stretches of homologous sequences flanking the DSB site. (C) The alternative end-joining (ALT-EJ) pathway anneals microhomologous sequences next to the DSB site. (D) In the homologous recombination $(\mathrm{HR})$ repair pathway, a recombinase (Rad51 and/or Dmc1 in most eukaryotes) utilizes a homologous template (i.e., sister chromatid) to faithfully repair the DSB.

TABLE 1 | HR DSB repair proteins, species, functions, and UniProtKB identifiers of human, yeast, and putative protozoan parasite homologs.

\begin{tabular}{|c|c|c|c|c|c|c|}
\hline Function & Homo sapiens & $\begin{array}{c}\text { Saccharomyces } \\
\text { cerevisiae }\end{array}$ & $\begin{array}{c}\text { Entamoeba } \\
\text { histolytica }\end{array}$ & $\begin{array}{c}\text { Trypanosoma } \\
\text { brucei }\end{array}$ & $\begin{array}{l}\text { Plasmodium } \\
\text { falciparum }\end{array}$ & $\begin{array}{c}\text { Leishmania } \\
\text { major }\end{array}$ \\
\hline \multirow[t]{3}{*}{ DSB End Resection } & MRE11 & Mre11 & C4LVX7 & Q8T8P1-? & A0A1C3KMQ6 & E9ADG7 \\
\hline & RAD50 & Rad50 & N9V1K1 & Q384J8 & C6KSQ6 & Q4Q8L7 \\
\hline & NSB1 & Xrs2 & $?$ & $?$ & $?$ & $?$ \\
\hline $\begin{array}{l}\text { Single-strand DNA } \\
\text { Binding }\end{array}$ & $\begin{array}{c}\text { RPA1-RPA2- } \\
\text { RPA3 }\end{array}$ & Rfa1-Rfa2-Rfa3 & $?-?-?$ & $?-?-?$ & $\begin{array}{l}\text { A0A1C3KN47/ } \\
\text { A0A1C3KQ72 }\end{array}$ & $?-?-?$ \\
\hline \multirow{2}{*}{$\begin{array}{l}\text { Recombinase } \\
\text { (Meiosis-specific) }\end{array}$} & RAD51 & Rad51 & Q86C17* & Q384KO* & Q8IIS8 & O61127 \\
\hline & DMC1 & Dmc1 & C4LTR6* & Q38E34* & A0A1C3КРB3 & 061128 \\
\hline \multirow[t]{7}{*}{ Accessory Proteins } & RAD54 & Rad54 & C4LVM6* & Q385M5 & Q8IAN4 & Q4QH75 \\
\hline & RAD54B & Rdh54 & N9TAM9 & $?$ & $?$ & $?$ \\
\hline & RAD55 & Rad55 & $?$ & $?$ & $?$ & $?$ \\
\hline & RAD57 & Rad57 & $?$ & $?$ & $?$ & $?$ \\
\hline & - & RAD59 & $?$ & $?$ & $?$ & $?$ \\
\hline & HOP2 & Hop2 & $?$ & $?$ & $?$ & $?$ \\
\hline & MND1 & Mnd1 & MЗTLCO & $?$ & C6S3J7 & Q4QAN2 \\
\hline $\begin{array}{l}\text { Single-Strand } \\
\text { Annealing }\end{array}$ & RAD52 & Rad52 & C4M197 & $?$ & $?$ & $?$ \\
\hline Mediator & BRCA2 & Rad52 & N9TLS7 & Q4GZF5 & $?$ & Q4QD38 \\
\hline
\end{tabular}

*Indicates characterized enzymes parasites.

?Indicates no known homolog.

\section{HOMOLOGOUS RECOMBINATION IN PROTOZOAN PARASITES}

Homologous recombination is essential in many protozoan parasites. These parasites utilize HR to adapt to diverse environmental conditions, evade host immune systems, and respond to DSBs (Deitsch et al., 1997; Bhattacharyya et al., 2004). Many of the core eukaryotic enzymes involved in HR are conserved among protozoan parasites (Table 1); however, the mechanisms of HR in many of these pathogens are largely unknown. Over the past few years, our understanding of HR in these organisms has increased from studies involving Trypanosoma, Leishmania, Plasmodium, and Entamoeba.

Homologous recombination plays a significant role in the survival of $T$. brucei, the protozoan parasite responsible for African trypanosomiasis (sleeping sickness). T. brucei generates 


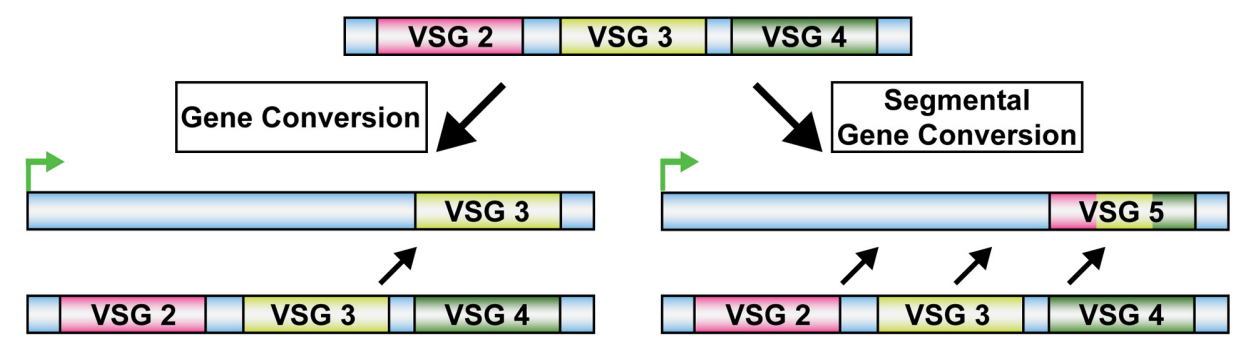

FIGURE 2 | Trypanosoma brucei Homologous Recombination VSG Switching. VSG Switching by homologous recombination in T. brucei occurs through gene conversion. This can occur by exchanging a VSG gene into a transcriptionally active site or through multiple VSG gene segment conversions into the transcriptionally active site.

antigenic variation through DNA recombination to evade the host immune system (McCulloch and Barry, 1999). In T. brucei, antigenic variation consists of repeatedly changing the expression of the Variant Surface Glycoprotein (VSG) genes that encode an outer protective coat of the parasite (Cross, 1975). T. brucei expresses more than 1000 VSG genes and pseudogenes one at a time (Borst, 2002; Marcello and Barry, 2007; McCulloch et al., 2015). One of the ways in which T. brucei can switch the expression of the VSG gene is through gene conversion of entire VSG genes or partial VSG pseudogenes into a transcriptionally active site using HR (Figure 2) (Borst, 2002; Marcello and Barry, 2007; Vink et al., 2012). It was shown that Rad51 was a major factor in this process, as null mutations of Rad51 in T. brucei ( $T b$ Rad51) led to a reduction in the VSG switching frequency (McCulloch and Barry, 1999). Moreover, mutations in genes involved in other DSB repair pathways (i.e., NHEJ) induced no change in VSG switching frequency (Conway et al., 2002a), indicating that VSG switching is likely dependent on $T b \operatorname{Rad} 51$. However, the frequency of VSG switching was reduced rather than eliminated in $T b \operatorname{Rad} 51$ mutants, suggesting that RAD51-mediated HR is not the only pathway for VSG switching (McCulloch and Barry, 1999; Conway et al., 2002a,b). Indeed, ALT-EJ also exists as an alternative pathway to $\mathrm{HR}$ in this pathogen (Conway et al., 2002b; Barnes and McCulloch, 2007; Glover et al., 2008, 2011). Furthermore, TbRad51 was reported to be important in response to DNA damage. When DSBs were induced in T. brucei, TbRad51 was shown to localize to subnuclear foci (Glover and Horn, 2012), and HR served as the predominant DSB repair pathway (Barnes and McCulloch, 2007; Glover et al., 2008).

Trypanosoma cruzi is a protozoan parasite that causes Chagas disease, or American trypanosomiasis. T. cruzi Rad51 (TcRad51) has a significant role in the response to and repair of DNA damage caused by ionizing radiation, as shown by in vivo analyses (Regis-da-Silva et al., 2006). Interestingly, the genomes of T. brucei and T. cruzi contain Dmcl genes with $65 \%$ and $70 \%$ identity to human DMC1, respectively (Proudfoot and McCulloch, 2006; Kelso et al., 2017). However, heterozygous and homozygous knockout DMC1 mutants $\left(D M C 1^{+/-}\right.$and $d m c 1^{-/-}$, respectively) in $T$. brucei were not sensitive to DNA damage, failed to impact VSG switching frequency, and had unaltered recombination efficiency, suggesting that $T b \mathrm{Dmcl}$ played no role in these processes (Proudfoot and McCulloch, 2006). These results were in direct contrast to the studies of T. brucei rad51-/- mutants (McCulloch and Barry, 1999; Conway et al., 2002b). Currently, there is no evidence to suggest that $T b \operatorname{Rad} 51$ compensates for the loss of $T b \mathrm{Dmcl}$, or vice versa. Future work will be necessary to reveal the biochemical underpinnings of Rad51 and Dmc1 recombinases from T. brucei and T. cruzi.

Leishmaniasis is a diverse disease characterized by three main forms: fever/anemia/weight loss, skin lesions, or mucous membrane destruction. Leishmaniasis is caused by the protozoan parasite Leishmania. Leishmania utilize HR to induce gene rearrangement events in response to oxidative stress, while HRmediated DNA amplification events serve as a mechanism for drug resistance (Beverley, 1991; Ouellette and Papadopoulou, 1993; Nathan and Shiloh, 2000; Ubeda et al., 2008, 2014; MonteNeto et al., 2015). Although HR is exploited in vitro to create null mutants of L. major for study (Cruz et al., 1991), the mechanisms of HR in L. major are largely unknown. There are a few studies demonstrating the existence of functional Rad51 in Leishmania. Namely, in vivo studies of L. major showed that the typically low levels of $\operatorname{Rad} 51(\operatorname{Lm} \operatorname{Rad} 51)$ expression increased in response to the DNA damaging agent phleomycin (McKean et al., 2001). Similarly, L. infantum Rad51 (LiRAD51) was highly expressed in response to phleomycin exposure, while the expression levels of the L. infantum ortholog of BRCA2 (LiBRCA2) were unchanged, similar to what has been reported in human cells (Lu et al., 2005). LiRAD51 and LiBRCA2 both localize to punctate foci in the nucleus; however, LiRAD51 fails to localize to the nucleus in LiBRCA2-deficient cells (Genois et al., 2012). Biochemically, $L m$ Rad51 ATP hydrolysis activity was stimulated by the presence of both ssDNA and dsDNA (McKean et al., 2001). Another biochemical study showed that LiRAD51 binds DNA-with an apparent higher affinity for ssDNA over dsDNA (Genois et al., 2012), and LiRAD51 can facilitate homologous DNA pairing (Genois et al., 2012). 
Furthermore, $L i B R C A 2$ was reported to load $L i R A D 51$ onto RPA coated ssDNA (Genois et al., 2012), similar to its human homolog (Jensen et al., 2010; Liu et al., 2010; Thorslund et al., 2010).

$P$. falciparum causes malaria in humans. The genome of P. falciparum contains many of the HR proteins (Table 1) (Gardner et al., 2002; Kirkman et al., 2014). In vivo studies using an exogenous HR reporter substrate with an inducible DSB site found that HR was the preferred DSB repair pathway when a homologous template was available, and during the more common haploid state, ALT-EJ was preferred (Kirkman et al., 2014). Upon exposure to the DNA damaging agent, methyl methanesulfonate (MMS), P. falciparum Rad51 (PfRad51) expression increased (Bhattacharyya and Kumar, 2003). Biochemical analysis of $P f \operatorname{Rad} 51$ showed that it hydrolyzed ATP and facilitated efficient DNA strand exchange (Bhattacharyya et al., 2005). PfRad51 required ATP binding but not hydrolysis to catalyze DNA strand exchange in vitro (Bhattacharyya et al., 2005), similar to human RAD51. In support of this notion, mutational analysis of the ATP binding motif of PfRad51 showed that $P f \operatorname{Rad} 51$ K143R significantly impacted the in vivo function of $P f \operatorname{Rad} 51$ (Roy et al., 2014). Furthermore, P. berghei (responsible for malaria in rodents) and S. cerevisiae expressing the $P f \operatorname{Rad} 51-\mathrm{K} 143 \mathrm{R}$ variant were found to be hypersensitive to MMS treatment (Roy et al., 2014).

In addition to the studies on $P f \operatorname{Rad} 51$, homologs of other HR proteins were shown to have similar functions and/or responses to DNA DSBs in Plasmodium as reported for their human counterparts. For example, Rad54 is a Rad51 stimulating protein, and $P f$ Rad54 similarly stimulated the homologous DNA pairing activity of $P f$ Rad51 (Gopalakrishnan and Kumar, 2013). BLM (Bloom syndrome protein) is a DNA helicase involved in DSB end-resection, and PfBlm possessed helicase activity (Rahman et al., 2016). The protein complex Mre11 (meiotic recombination 11), Rad50 (radiation-sensitive 50), and NBS1 (involved in Nijmegen breakage syndrome) recognizes and endresects DNA DSBs, and $P f$ Mre 11 demonstrated nuclease activity and interacted with PfRad50 (Badugu et al., 2015). Lastly, diploid zygotes of Plasmodium undergo meiosis in the insect vector to produce haploid cells, a state in which Dmcl becomes relevant. One study in $P$. berghei null for $d m c 1$ demonstrated that $P b \mathrm{Dmcl}$ was essential for proper oocyst development (Mlambo et al., 2012). PbDmcl null cells were also shown to be highly sensitive to a DNA damaging agent (Mlambo et al., 2012).

Entamoeba histolytica is the parasite responsible for amoebic dysentery and amoebic liver abscess. The life cycle of E. histolytica consists of a tetra-nucleated cyst that is transmitted through water or food sources by fecal contamination (Figure 3). When ingested by the host, the mature, tetra-nucleated cyst can undergo excystation in the small intestine resulting in the release of eight trophozoites that can colonize the large intestine. Trophozoites multiply by binary fission and produce multinucleated cysts through the process of encystation (Dobell, 1928; Cleveland and Sanders, 1930; Ratcliffe and Geiman, 1934; Koushik et al., 2014). There is ample support for $\mathrm{HR}$ in E. histolytica. For example, the genome of E. histolytica contains the majority of the HR core genes (Table 1) (Bhattacharya et al., 2000). Also, genome duplication events, unscheduled gene amplification events, and genetic rearrangements are reported in E. histolytica and thought to be mediated by HR (Orozco et al., 1988; Baez-Camargo et al., 1996; Bhattacharya et al., 2000; Mukherjee et al., 2008). The first study on $\mathrm{HR}$ in E. histolytica monitored the response of E. histolytica to UV-C radiation, which is known to cause DNA damage (Lopez-Casamichana et al., 2008). Many hallmarks of HR were observed upon DNA damage induction in E. histolytica: the histone $\mathrm{H} 2 \mathrm{AX}$ was phosphorylated (indicating DNA damage), cell survivability was not affected (indicating DSB repair occurred), the core HR genes were differentially expressed in response to irradiation, EhRad51 expression (mRNA and protein) peaked rapidly in response to DNA damage, and EhRad51 formed nuclear foci in response to DNA damage (Lopez-Casamichana et al., 2008; Charcas-Lopez Mdel et al., 2014). During growth stresses (heat shock, oxygen stress, serum starvation) and in response to DNA damage (UV irradiation), inverted repeat sequences located either on a plasmid or in the E. histolytica genome underwent recombination (Singh et al., 2013). This represents the first direct evidence for $\mathrm{HR}$ in E. histolytica. Biochemically, Lopez-Casamichana et al. (2008) showed that partially purified EhRad51 bound DNA and facilitated homologous DNA pairing (Lopez-Casamichana et al., 2008). The findings were recently extended by a report that demonstrated EhRad51 was not only capable of binding DNA and forming D-loops, but that EhRad51 hydrolyzed ATP, formed presynaptic filaments, and catalyzed DNA strand exchange over thousands of base pairs (Kelso et al., 2016). Although E. histolytica reproduce asexually through binary fission, the occurrence of meiosis in E. histolytica was first proposed to occur during encystation based on increased expression of meiosis-specific genes (Ehrenkaufer et al., 2013). Additional support for this notion came from monitoring the expression of meiosis-specific genes in the reptilian amoebozoa parasite, E. invadens during encystation (Singh et al., 2013). More recently, biochemical analysis of EhDmcl demonstrated the enzyme hydrolyzed ATP in the presence of DNA, bound DNA, formed nucleoprotein filaments, and catalyzed homologous DNA pairing and DNA strand exchange (Kelso et al., 2015). Taken together, the evidence suggests that mitotic and meiotic HR occur and are likely important for encystation in Entamoeba (Figure 3).

Currently, the roles of other DSB repair pathways in protozoan parasites are largely uncharacterized. As mentioned above, ALTEJ has been reported for some parasites, but the mechanisms remain elusive (Conway et al., 2002b; Barnes and McCulloch, 2007; Glover et al., 2008, 2011; Kirkman et al., 2014). As for SSA, although many genomes of protozoan parasites contain the RAD52 gene, T. brucei is the only pathogen to demonstrate a potential for SSA (Glover and Horn, 2009). Lastly, the genomes of some parasites, like T. brucei and E. histolytica, contain factors necessary for NHEJ (e.g., Ku); however, many of the key components of this pathway are missing such as DNA ligase IV and XRCC4 homologs (Burton et al., 2007). In fact, T. brucei Ku was shown to have a role in telomere length maintenance, but DSB repair occurred independent of $\mathrm{Ku}$ (Conway et al., 2002a). 


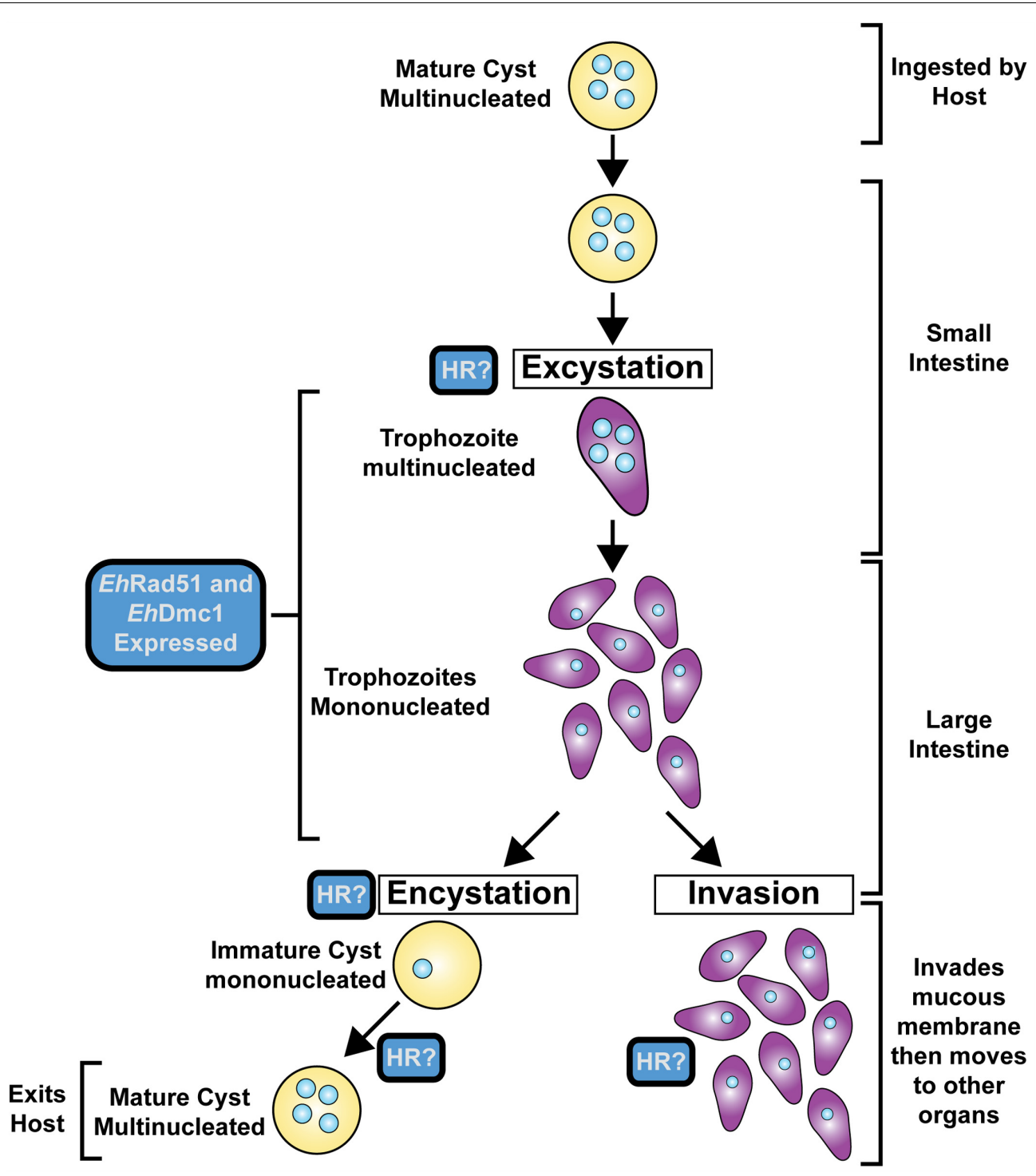

FIGURE 3 | Life Cycle of Entamoeba histolytica. The human host ingests the mature cyst. The cyst moves to the small intestine where excystation occurs, resulting in the release of multiple trophozoites that migrate to the large intestine. Trophozoites either invade the mucous membrane of the host or encyst and exit the host for future infections.

\section{TARGETING HOMOLOGOUS RECOMBINATION WITH SMALL MOLECULE INHIBITORS}

Studies of small molecule inhibitors targeting the proteins involved in HR have increased in recent years (Bryant et al., 2005; Ishida et al., 2009; Huang et al., 2011, 2012; Takaku et al., 2011; Budke et al., 2012, 2013; Huang and Mazin, 2014; Normand et al., 2014). Many current anticancer therapies, such as irradiation and chemotherapy, are designed to induce DNA damage and provoke an apoptotic response. By targeting major HR enzymes like RAD51, RAD54, and BRCA2, the susceptibility of cancer cells could increase when combined with other DNAdamaging therapies. An analogous argument could be made for the targeting of enzymes involved in HR in protozoan parasites, which rely on HR for diverse environmental adaptations, host immune evasion, and drug resistances (Deitsch et al., 1997; Bhattacharyya et al., 2004).

RAD51 serves as a reasonable drug target for protozoan parasites because it is important for the repair of damaged DNA by HR. However, for RAD51 to be an effective target for small molecule inhibitors in these pathogens, it is essential to develop inhibitors that are species-specific, since RAD51 is also ubiquitously expressed in the human host. Currently, there is limited structural information about the recombinases, which hinders the potential for structure-based drug design. Due to the availability of purified recombinases, high throughput screens could provide a powerful platform for identifying potential 


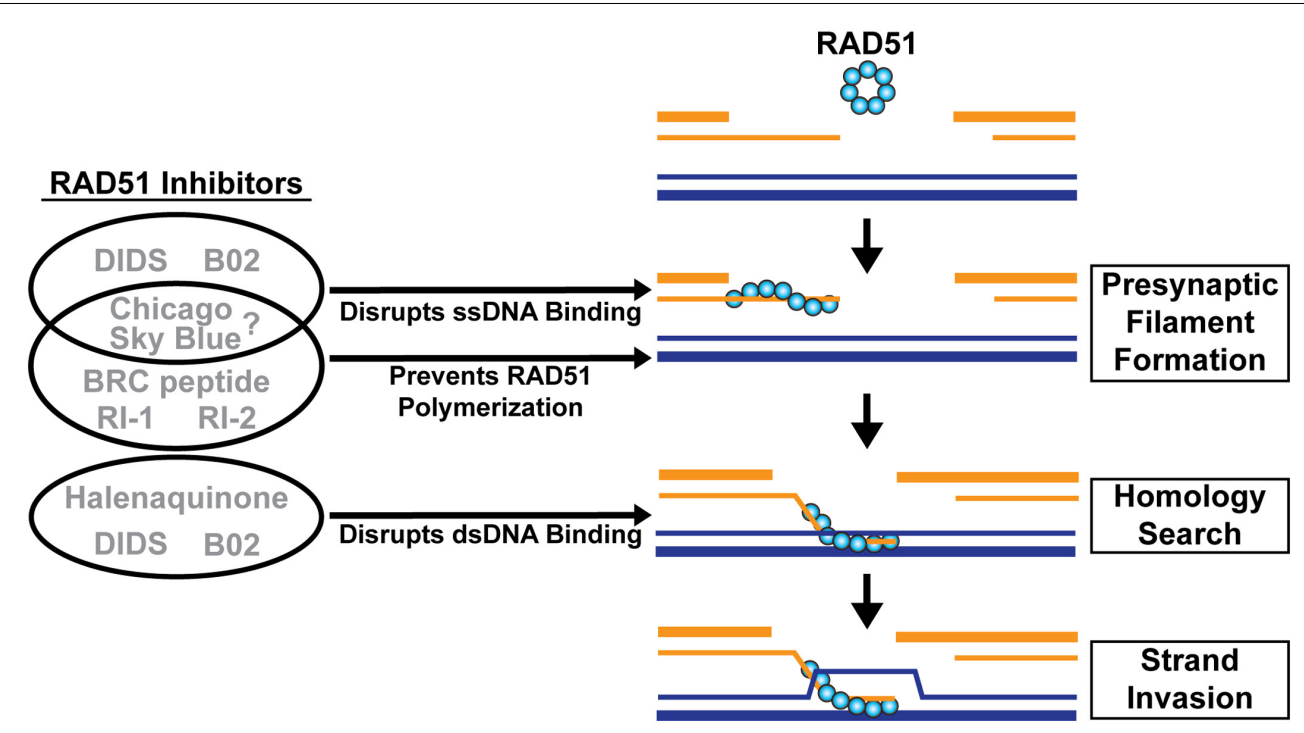

FIGURE 4 | Inhibitors of human RAD51. RAD51 inhibitors can prevent RAD51 from self-associating or from binding ssDNA and/or dsDNA. DIDS and B02 disrupt ssDNA and dsDNA binding. Chicago Sky Blue prevents filament formation but it is unknown if this is through inhibition of ssDNA binding or RAD51 polymerization. RI-1 and RI-2 block RAD51 polymerization binding sites, and the BRC peptide binds filament-dissociated RAD51 monomers, leading to inhibition. Halenaquinone disrupts the dsDNA binding of RAD51.

compounds that demonstrate efficient inhibition of parasitic recombinases but not human recombinases. Additionally, there are many well-characterized biochemical assays that could act as secondary analyses to aid in the understanding of mechanisms of inhibition. On a related note, recent studies have utilized small molecules, previously demonstrated to inhibit human RAD51, to target the activities of the E. histolytica recombinases (Kelso et al., 2015, 2016). The small molecule 4,4' diisothiocyanostilbene-2,2'-disulfonic acid (DIDS) was found to directly interact with human RAD51 and interfere with its ability to bind ssDNA and dsDNA (Figure 4) (Ishida et al., 2009). By interfering with the ability of RAD51 to bind DNA, DIDS decreases the presynaptic filament formation of RAD51, which is essential for DNA strand exchange, D-loop formation, and ATP hydrolysis (Ishida et al., 2009). This small molecule was also shown to disrupt the recombinase activities of both EhDmc1 and EhRad51 in vitro (Kelso et al., 2015, 2016). Notably, encystation was significantly hindered in E. invadens when DIDS was present (Kelso et al., 2016). Despite its efficacy in these pathogens, DIDS exhibits high toxicity for human cells, complicating its use as a potential anti-pathogen treatment (Ishida et al., 2009). Interestingly, in the same study by Kelso et al. (2016), another human RAD51 small molecule inhibitor, B02, was tested. B02 was reported to disrupt RAD51 binding to ssDNA during presynaptic filament formation. In addition, B02 disrupts the subsequent binding of dsDNA to the RAD51-ssDNA complex (Figure 4) (Huang et al., 2011). As a result, B02 inhibits human RAD51-mediated DNA strand exchange and D-loop formation activities (Huang et al., 2011). In vivo studies revealed that $\mathrm{B} 02$ also prevents RAD51 foci formation at DSBs and effectively sensitizes human cells to DNA damage by the interstrand crosslinking agents cisplatin and mitomycin-C and to PARP1 inhibitors, which target the PARP1 system responsible for repair of ssDNA breaks (Huang et al., 2011, 2012; Huang and Mazin, 2014). When tested with EhDmc1 and EhRad51, B02 failed to disrupt their DNA strand exchange activity at concentrations that inhibited human RAD51 (Kelso et al., 2016). This was the first study to demonstrate species selectivity by small molecule inhibitors of human and protozoan recombinases. Importantly, these findings provide evidence that inhibitors could potentially be tailored to target specific recombinases.

There are additional small molecule inhibitors reported to disrupt human RAD51 activities that have not been characterized for their efficacy against protozoan recombinases. Halenaquinone disrupts the ability of RAD51 to bind dsDNA regardless of the presence of ssDNA (Takaku et al., 2011). This suggests that the compound binds to the dsDNA binding site, severely compromising the ability of RAD51 to catalyze homologous DNA pairing (Figure 4). Thus, in vivo studies demonstrated that halenaquinone suppressed the formation and retention of RAD51 foci at DSB sites (Takaku et al., 2011). Although halenaquinone interacts with RAD51 in a manner similar to DIDS, it does not exhibit the same level of toxicity for human cells (Takaku et al., 2011).

RI-1 is a compound that was shown to inhibit human RAD51 presynaptic filament formation by irreversibly binding to cysteine-319 at the oligomerization interface (Figure 4) (Budke et al., 2012). By inhibiting monomer-monomer interaction, RI-1 prevents polymerization of the RAD51 filament onto ssDNA and consequently inhibits the assembly of RAD51 foci in vivo (Budke et al., 2012). Additionally, RI-1 was shown to sensitize cancer cells to mitomycin-C, a compound that induces DNA 
damage via cross-linking. RI-1 reacts with thiol groups (hence conjugation to cysteine-319) and therefore has the potential for off-target effects. Thus, RI-2, a derivative of RI-1, was developed to inhibit RAD51 via the same mechanism but in a reversible capacity in order to reduce the likelihood of serious off-target binding effects (Figure 4) (Budke et al., 2013). RI-2 was also shown to have a longer half-life than RI-1 and is a functional inhibitor of RAD51 in human cells. However, due to the nature of its reversible binding to the cysteine-319 of RAD51, RI-2 requires higher concentrations to achieve the same efficacy as the irreversibly bound RI-1 (Budke et al., 2013).

Chicago Sky Blue (CSB) is a potent inhibitor of human RAD51 activity, with sufficient inhibition observed in the $400 \mathrm{nM}$ range (Normand et al., 2014). CSB inhibits D-loop formation and DNA strand exchange activity of RAD51 by preventing filament assembly of RAD51 onto ssDNA, effectively inhibiting its HR activities (Normand et al., 2014). However, it is unclear if the suppression of RAD51-ssDNA binding occurs by interfering with the ability of RAD51 to bind ssDNA (as seen with DIDS) or by disruption of protein polymerization onto ssDNA via binding at the protein-protein interface (as seen with RI-1 and RI-2) (Figure 4). Importantly, CSB exhibits the lowest $\mathrm{IC}_{50}(400 \mathrm{nM})$ of the RAD51 inhibitory compounds, making it a potent RAD51 inhibitor with significant clinical potential (Normand et al., 2014).

Lastly, a short peptide of the BRC4 repeat from human BRCA2 was shown to dissociate the RAD51 filament by binding to the filament-dissociated monomers of RAD51 thereby rendering RAD51 inactive through sequestration (Figure 4) (Nomme et al., 2008).

Given that RAD51 is highly conserved among eukaryotes and is ubiquitously expressed, other HR proteins might serve as alternative therapeutic targets. Currently, HR in protozoan parasites is an emerging field, and therefore, the enzymes involved are not well understood. As this field develops, new HR targets that are essential and specific to parasites may emerge. Consequently, identifying if these exist in protozoan parasites would be valuable for potential pathogenic targets.

\section{CONCLUSION AND PERSPECTIVES}

Homologous recombination is an essential DNA repair process. Dysfunction of this pathway can challenge the viability and fecundity of an organism. The majority of the HR repair machinery is highly conserved among eukaryotes, including RAD51. RAD51 is responsible for DNA strand exchange and homology search via the formation of a nucleoprotein filament on ssDNA overhangs after end resection of a DSB. As a result of its central role in HR, there is merit for considering RAD51 as potential therapeutic targets in human pathogens. Presently, there is no evidence to suggest that protozoa can use the repair machinery of their host; thus, targeting protozoan RAD51 remains feasible. Interestingly, one study demonstrated the ability of a mammalian accessory protein, murine Hop2-Mnd1, to stimulate the recombinase activities of EhRad51, so it is reasonable to consider the possibility. Further studies will be necessary to examine this hypothesis. Many of the RAD51 inhibitors reviewed here (DIDS, Halenaquinone, B02, CBS, BRC peptide) impede the interaction between RAD51 and DNA. As this function is essential for RAD51 filament formation and localization to DSB sites, it is an effective target to achieve inhibition of RAD51 activity in HR. Another class of RAD51 inhibitors (including RI-1 and RI-2) disrupt presynaptic filament formation by physically preventing RAD51 polymerization onto ssDNA. Again, inhibition of RAD51 at this early HR stage abolishes repair of DSBs, and therefore can lead to cell death. The ability to disrupt RAD51 in parasites and not inhibit human RAD51 will be critical for the development of new species-specific small molecule inhibitors. Analysis of the targeted impact of some of these molecules on EhRad51 versus human RAD51 suggests that there is some degree of variance between the homologs that allows molecule specificity (note the specificity of B02 for human RAD51). Since protozoan parasites rely on $\mathrm{HR}$ for host immune evasion and drug resistance, targeting the major enzymes involved in the HR pathway could lead to new therapeutic intervention strategies. For example, under stressful environmental conditions, E. histolytica can undergo encystation for survival, which involves multiple rounds of DNA replication that produces a polyploid cyst. It has been suggested that $H R$ plays a substantial role in encystation in E. histolytica, which has been modeled in the related reptilian parasite, E. invadens. Thus, inhibition of Entamoeba HR via EhRad51 small molecule inhibitors could limit Entamoeba pathogenicity by obstructing the encystation pathways. Future studies are necessary to determine the efficacy of these human recombinase inhibitors on the survivability of protozoan parasites. Additionally, small molecule inhibitor studies on human RAD51 could provide a platform for future compound design against the RAD51 recombinase from other organisms.

\section{AUTHOR CONTRIBUTIONS}

AK and MS conceived and designed the study. AK, SW, AL, and MS wrote and edited the manuscript. All authors approved the content of the manuscript for accuracy.

\section{FUNDING}

This work was supported in part by the Clemson University Creative Inquiry Program (SW and AL), Departmental Honors Research Grants from the Calhoun Honors College, Clemson University (SW and AL) and by the National Institutes of Health R01GM098510 (MS). Processing charges for this publication generously supported by the Clemson University Libraries' Open Access Publishing Fund.

\section{ACKNOWLEDGMENT}

The authors would like to thank Dr. Lesly Temesvari for the helpful comments on the manuscript. 


\section{REFERENCES}

Badugu, S. B., Nabi, S. A., Vaidyam, P., Laskar, S., Bhattacharyya, S., and Bhattacharyya, M. K. (2015). Identification of Plasmodium falciparum DNA repair protein Mre11 with an evolutionarily conserved nuclease function. PLOS ONE 10:e0125358. doi: 10.1371/journal.pone.0125358

Baez-Camargo, M., Gharaibeh, R., Riveron, A. M., De La Cruz Hernandez, F., Luna, J. P., Gariglio, P., et al. (1996). Gene amplification in Entamoeba histolytica. Invasion Metastasis 16, 269-279.

Barnes, R. L., and McCulloch, R. (2007). Trypanosoma brucei homologous recombination is dependent on substrate length and homology, though displays a differential dependence on mismatch repair as substrate length decreases. Nucleic Acids Res. 35, 3478-3493. doi: 10.1093/nar/gkm249

Betermier, M., Bertrand, P., and Lopez, B. S. (2014). Is non-homologous endjoining really an inherently error-prone process? PLOS Genet. 10:e1004086. doi: 10.1371/journal.pgen.1004086

Beverley, S. M. (1991). Gene amplification in Leishmania. Annu. Rev. Microbiol. 45, 417-444. doi: 10.1146/annurev.mi.45.100191.002221

Bhargava, R., Onyango, D. O., and Stark, J. M. (2016). Regulation of single-strand annealing and its role in genome maintenance. Trends Genet. 32, 566-575. doi: 10.1016/j.tig.2016.06.007

Bhattacharya, A., Satish, S., Bagchi, A., and Bhattacharya, S. (2000). The genome of Entamoeba histolytica. Int. J. Parasitol. 30, 401-410. doi: 10.1016/S00207519(99)00189-7

Bhattacharyya, M. K., Bhattacharyya Nee Deb, S., Jayabalasingham, B., and Kumar, N. (2005). Characterization of kinetics of DNA strand-exchange and ATP hydrolysis activities of recombinant PfRad51, a Plasmodium falciparum recombinase. Mol. Biochem. Parasitol. 139, 33-39. doi: 10.1016/j.molbiopara. 2004.09.007

Bhattacharyya, M. K., and Kumar, N. (2003). Identification and molecular characterisation of DNA damaging agent induced expression of Plasmodium falciparum recombination protein PfRad51. Int. J. Parasitol. 33, 1385-1392. doi: $10.1016 / S 0020-7519(03) 00212-1$

Bhattacharyya, M. K., Norris, D. E., and Kumar, N. (2004). Molecular players of homologous recombination in protozoan parasites: implications for generating antigenic variation. Infect. Genet. Evol. 4, 91-98. doi: 10.1016/j.meegid.2004. 01.008

Binz, S. K., Sheehan, A. M., and Wold, M. S. (2004). Replication protein A phosphorylation and the cellular response to DNA damage. DNA Repair 3, 1015-1024. doi: 10.1016/j.dnarep.2004.03.028

Bishop, D. K. (2012). Rad51, the lead in mitotic recombinational DNA repair, plays a supporting role in budding yeast meiosis. Cell Cycle 11, 4105-4106. doi: $10.4161 / c c .22396$

Bishop, D. K., Park, D., Xu, L., and Kleckner, N. (1992). DMC1: a meiosisspecific yeast homolog of $E$. coli recA required for recombination, synaptonemal complex formation, and cell cycle progression. Cell 69, 439-456. doi: 10.1016/ 0092-8674(92)90446-J

Borst, P. (2002). Antigenic variation and allelic exclusion. Cell 109, 5-8. doi: 10. 1016/S0092-8674(02)00711-0

Bryant, H. E., Schultz, N., Thomas, H. D., Parker, K. M., Flower, D., Lopez, E., et al. (2005). Specific killing of BRCA2-deficient tumours with inhibitors of poly(ADP-ribose) polymerase. Nature 434, 913-917. doi: 10.1038/nature03443

Budke, B., Kalin, J. H., Pawlowski, M., Zelivianskaia, A. S., Wu, M., Kozikowski, A. P., et al. (2013). An optimized RAD51 inhibitor that disrupts homologous recombination without requiring Michael acceptor reactivity. J. Med. Chem. 56, 254-263. doi: 10.1021/jm301565b

Budke, B., Logan, H. L., Kalin, J. H., Zelivianskaia, A. S., Cameron Mcguire, W., Miller, L. L., et al. (2012). RI-1: a chemical inhibitor of RAD51 that disrupts homologous recombination in human cells. Nucleic Acids Res. 40, 7347-7357. doi: $10.1093 /$ nar/gks353

Burton, P., Mcbride, D. J., Wilkes, J. M., Barry, J. D., and Mcculloch, R. (2007). Ku heterodimer-independent end joining in Trypanosoma brucei cell extracts relies upon sequence microhomology. Eukaryot. Cell 6, 1773-1781. doi: 10.1128/EC. 00212-07

Charcas-Lopez Mdel, S., Garcia-Morales, L., Pezet-Valdez, M., LopezCamarillo, C., Zamorano-Carrillo, A., and Marchat, L. A. (2014). Expression of EhRAD54, EhRAD51, and EhBLM proteins during DNA repair by homologous recombination in Entamoeba histolytica. Parasite 21:7. doi: 10.1051/parasite/2014006

Chi, P., San Filippo, J., Sehorn, M. G., Petukhova, G. V., and Sung, P. (2007). Bipartite stimulatory action of the Hop2-Mnd1 complex on the Rad51 recombinase. Genes Dev. 21, 1747-1757. doi: 10.1101/gad.1563007

Cleveland, L. R., and Sanders, E. P. (1930). The production of bacteria-free amoebic abscesses in the liver of cats and observations on the amoebae in various media with and without bacteria. Science 72, 149-151. doi: 10.1126/science.72.18 58.149

Conway, C., Mcculloch, R., Ginger, M. L., Robinson, N. P., Browitt, A., and Barry, J. D. (2002a). Ku is important for telomere maintenance, but not for differential expression of telomeric VSG genes, in African trypanosomes. J. Biol. Chem. 277, 21269-21277.

Conway, C., Proudfoot, C., Burton, P., Barry, J. D., and Mcculloch, R. (2002b). Two pathways of homologous recombination in Trypanosoma brucei. Mol. Microbiol. 45, 1687-1700.

Cross, G. A. (1975). Identification, purification and properties of clone-specific glycoprotein antigens constituting the surface coat of Trypanosoma brucei. Parasitology 71, 393-417. doi: 10.1017/S003118200004717X

Cruz, A., Coburn, C. M., and Beverley, S. M. (1991). Double targeted gene replacement for creating null mutants. Proc. Natl. Acad. Sci. U.S.A. 88, 7170-7174. doi: 10.1073/pnas.88.16.7170

Deitsch, K. W., Moxon, E. R., and Wellems, T. E. (1997). Shared themes of antigenic variation and virulence in bacterial, protozoal, and fungal infections. Microbiol. Mol. Biol. Rev. 61, 281-293.

Dobell, C. (1928). Researches on the intestinal protozoa of monkeys and man. Parasitology 20, 357-412. doi: 10.1017/S003118200001177X

Ehrenkaufer, G. M., Weedall, G. D., Williams, D., Lorenzi, H. A., Caler, E., Hall, N., et al. (2013). The genome and transcriptome of the enteric parasite Entamoeba invadens, a model for encystation. Genome Biol. 14:R77. doi: 10.1186/gb-201314-7-r77

Gardner, M. J., Hall, N., Fung, E., White, O., Berriman, M., Hyman, R. W., et al. (2002). Genome sequence of the human malaria parasite Plasmodium falciparum. Nature 419, 498-511. doi: 10.1038/nature01097

Genois, M. M., Mukherjee, A., Ubeda, J. M., Buisson, R., Paquet, E., Roy, G., et al. (2012). Interactions between BRCA2 and RAD51 for promoting homologous recombination in Leishmania infantum. Nucleic Acids Res. 40, 6570-6584. doi: $10.1093 / \mathrm{nar} / \mathrm{gks} 306$

Ghezraoui, H., Piganeau, M., Renouf, B., Renaud, J. B., Sallmyr, A., Ruis, B., et al. (2014). Chromosomal translocations in human cells are generated by canonical nonhomologous end-joining. Mol. Cell. 55, 829-842. doi: 10.1016/ j.molcel.2014.08.002

Glover, L., and Horn, D. (2009). Site-specific DNA double-strand breaks greatly increase stable transformation efficiency in Trypanosoma brucei. Mol. Biochem. Parasitol. 166, 194-197. doi: 10.1016/j.molbiopara.2009.03.010

Glover, L., and Horn, D. (2012). Trypanosomal histone gammaH2A and the DNA damage response. Mol. Biochem. Parasitol. 183, 78-83. doi: 10.1016/j. molbiopara.2012.01.008

Glover, L., Jun, J., and Horn, D. (2011). Microhomology-mediated deletion and gene conversion in African trypanosomes. Nucleic Acids Res. 39, 1372-1380. doi: $10.1093 / \mathrm{nar} / \mathrm{gkq} 981$

Glover, L., Mcculloch, R., and Horn, D. (2008). Sequence homology and microhomology dominate chromosomal double-strand break repair in African trypanosomes. Nucleic Acids Res. 36, 2608-2618. doi: 10.1093/nar/gkn104

Gopalakrishnan, A. M., and Kumar, N. (2013). Opposing roles for two molecular forms of replication protein A in Rad51-Rad54-mediated DNA recombination in Plasmodium falciparum. MBio 4:e00252-13. doi: 10.1128/mBio.00252-13

Huang, F., and Mazin, A. V. (2014). A small molecule inhibitor of human RAD51 potentiates breast cancer cell killing by therapeutic agents in mouse xenografts. PLOS ONE 9:e100993. doi: 10.1371/journal.pone.0100993

Huang, F., Mazina, O. M., Zentner, I. J., Cocklin, S., and Mazin, A. V. (2012). Inhibition of homologous recombination in human cells by targeting RAD51 recombinase. J. Med. Chem. 55, 3011-3020. doi: 10.1021/jm201173g

Huang, F., Motlekar, N. A., Burgwin, C. M., Napper, A. D., Diamond, S. L., and Mazin, A. V. (2011). Identification of specific inhibitors of human RAD51 recombinase using high-throughput screening. ACS Chem. Biol. 6, 628-635. doi: $10.1021 / \mathrm{cb} 100428 \mathrm{c}$ 
Hunter, N. (2015). Meiotic recombination: the essence of heredity. Cold Spring Harb. Perspect. Biol. 7:a016618. doi: 10.1101/cshperspect.a016618

Ishida, T., Takizawa, Y., Kainuma, T., Inoue, J., Mikawa, T., Shibata, T., et al. (2009). DIDS, a chemical compound that inhibits RAD51-mediated homologous pairing and strand exchange. Nucleic Acids Res. 37, 3367-3376. doi: 10.1093/ nar/gkp200

Jensen, R. B., Carreira, A., and Kowalczykowski, S. C. (2010). Purified human BRCA2 stimulates RAD51-mediated recombination. Nature 467, 678-683. doi: 10.1038/nature09399

Kelso, A. A., Goodson, S. D., Chavan, S., Say, A. F., Turchick, A., Sharma, D., et al. (2016). Characterization of the recombination activities of the Entamoeba histolytica Rad51 recombinase. Mol. Biochem. Parasitol. 210, 71-84. doi: 10. 1016/j.molbiopara.2016.09.001

Kelso, A. A., Goodson, S. D., Temesvari, L. A., and Sehorn, M. G. (2017). Data on Rad51 amino acid sequences from higher and lower eukaryotic model organisms and parasites. Data Brief 10, 364-368. doi: 10.1016/j.dib.2016.12.002

Kelso, A. A., Say, A. F., Sharma, D., Ledford, L. L., Turchick, A., Saski, C. A., et al. (2015). Entamoeba histolytica Dmcl catalyzes homologous DNA pairing and strand exchange that is stimulated by calcium and Hop2-Mnd1. PLOS ONE 10:e0139399. doi: 10.1371/journal.pone.0139399

Kirkman, L. A., Lawrence, E. A., and Deitsch, K. W. (2014). Malaria parasites utilize both homologous recombination and alternative end joining pathways to maintain genome integrity. Nucleic Acids Res. 42, 370-379. doi: 10.1093/nar/ gkt881

Koushik, A. B., Welter, B. H., Rock, M. L., and Temesvari, L. A. (2014). A genomewide overexpression screen identifies genes involved in the phosphatidylinositol 3-kinase pathway in the human protozoan parasite Entamoeba histolytica. Eukaryot. Cell 13, 401-411. doi: 10.1128/EC.00329-13

Lieber, M. R. (2010). The mechanism of double-strand DNA break repair by the nonhomologous DNA end-joining pathway. Annu. Rev. Biochem. 79, 181-211. doi: 10.1146/annurev.biochem.052308.093131

Liu, J., Doty, T., Gibson, B., and Heyer, W. D. (2010). Human BRCA2 protein promotes RAD51 filament formation on RPA-covered single-stranded DNA. Nat. Struct. Mol. Biol. 17, 1260-1262. doi: 10.1038/nsmb.1904

Lopez-Casamichana, M., Orozco, E., Marchat, L. A., and Lopez-Camarillo, C. (2008). Transcriptional profile of the homologous recombination machinery and characterization of the EhRAD51 recombinase in response to DNA damage in Entamoeba histolytica. BMC Mol. Biol. 9:35. doi: 10.1186/1471-2199-9-35

Lu, H., Guo, X., Meng, X., Liu, J., Allen, C., Wray, J., et al. (2005). The BRCA2interacting protein BCCIP functions in RAD51 and BRCA2 focus formation and homologous recombinational repair. Mol. Cell. Biol. 25, 1949-1957. doi: 10.1128/MCB.25.5.1949-1957.2005

Malu, S., Malshetty, V., Francis, D., and Cortes, P. (2012). Role of non-homologous end joining in $\mathrm{V}(\mathrm{D}) \mathrm{J}$ recombination. Immunol. Res. 54, 233-246. doi: 10.1007/ s12026-012-8329-z

Marcello, L., and Barry, J. D. (2007). Analysis of the VSG gene silent archive in Trypanosoma brucei reveals that mosaic gene expression is prominent in antigenic variation and is favored by archive substructure. Genome Res. 17, 1344-1352. doi: $10.1101 /$ gr.6421207

Mason, J. M., Dusad, K., Wright, W. D., Grubb, J., Budke, B., Heyer, W. D., et al. (2015). RAD54 family translocases counter genotoxic effects of RAD51 in human tumor cells. Nucleic Acids Res. 43, 3180-3196. doi: 10.1093/nar/gkv175

Matos, J., and West, S. C. (2014). Holliday junction resolution: regulation in space and time. DNA Repair 19, 176-181. doi: 10.1016/j.dnarep.2014.03.013

McCulloch, R., and Barry, J. D. (1999). A role for RAD51 and homologous recombination in Trypanosoma brucei antigenic variation. Genes Dev. 13, 2875-2888. doi: 10.1101/gad.13.21.2875

McCulloch, R., Morrison, L. J., and Hall, J. P. (2015). DNA recombination strategies during antigenic variation in the African trypanosome. Microbiol. Spectr. 3, 1-25. doi: 10.1128/microbiolspec.MDNA3-0016-2014

McKean, P. G., Keen, J. K., Smith, D. F., and Benson, F. E. (2001). Identification and characterisation of a RAD51 gene from Leishmania major. Mol. Biochem. Parasitol. 115, 209-216. doi: 10.1016/S0166-6851(01)00288-2

Mehta, A., and Haber, J. E. (2014). Sources of DNA double-strand breaks and models of recombinational DNA repair. Cold Spring Harb. Perspect. Biol. 6:a016428. doi: 10.1101/cshperspect.a016428

Mimori, T., and Hardin, J. A. (1986). Mechanism of interaction between Ku protein and DNA. J. Biol. Chem. 261, 10375-10379.
Mlambo, G., Coppens, I., and Kumar, N. (2012). Aberrant sporogonic development of Dmc1 (a meiotic recombinase) deficient Plasmodium berghei parasites. PLOS ONE 7:e52480. doi: 10.1371/journal.pone.0052480

Monte-Neto, R., Laffitte, M. C., Leprohon, P., Reis, P., Frezard, F., and Ouellette, M. (2015). Intrachromosomal amplification, locus deletion and point mutation in the aquaglyceroporin AQP1 gene in antimony resistant Leishmania (Viannia) guyanensis. PLOS Negl. Trop. Dis. 9:e0003476. doi: 10.1371/journal.pntd. 0003476

Moore, C. W. (1978). Responses of radiation-sensitive mutants of Saccharomyces cerevisiae to lethal effects of bleomycin. Mutat. Res. 51, 165-180. doi: 10.1016/ S0027-5107(78)80016-5

Morales, M. E., White, T. B., Streva, V. A., Defreece, C. B., Hedges, D. J., and Deininger, P. L. (2015). The contribution of alu elements to mutagenic DNA double-strand break repair. PLOS Genet. 11:e1005016. doi: 10.1371/journal. pgen.1005016

Morrical, S. W. (2015). DNA-pairing and annealing processes in homologous recombination and homology-directed repair. Cold Spring Harb. Perspect. Biol. 7:a016444. doi: 10.1101/cshperspect.a016444

Moynahan, M. E., and Jasin, M. (2010). Mitotic homologous recombination maintains genomic stability and suppresses tumorigenesis. Nat. Rev. Mol. Cell Biol. 11, 196-207. doi: 10.1038/nrm2851

Mukherjee, C., Clark, C. G., and Lohia, A. (2008). Entamoeba shows reversible variation in ploidy under different growth conditions and between life cycle phases. PLOS Negl. Trop. Dis. 2:e281. doi: 10.1371/journal.pntd. 0000281

Nathan, C., and Shiloh, M. U. (2000). Reactive oxygen and nitrogen intermediates in the relationship between mammalian hosts and microbial pathogens. Proc. Natl. Acad. Sci. U.S.A. 97, 8841-8848. doi: 10.1073/pnas.97.16.8841

Nomme, J., Takizawa, Y., Martinez, S. F., Renodon-Corniere, A., Fleury, F., Weigel, P., et al. (2008). Inhibition of filament formation of human Rad51 protein by a small peptide derived from the BRC-motif of the BRCA2 protein. Genes Cells 13, 471-481. doi: 10.1111/j.1365-2443.2008.01180.x

Normand, A., Riviere, E., and Renodon-Corniere, A. (2014). Identification and characterization of human Rad51 inhibitors by screening of an existing drug library. Biochem. Pharmacol. 91, 293-300. doi: 10.1016/j.bcp.2014.07.033

Orozco, E., Solis, F. J., Dominguez, J., Chavez, B., and Hernandez, F. (1988). Entamoeba histolytica: cell cycle and nuclear division. Exp. Parasitol. 67, 85-95. doi: 10.1016/0014-4894(88)90011-2

Ouellette, M., and Papadopoulou, B. (1993). Mechanisms of drug resistance in Leishmania. Parasitol. Today 9, 150-153. doi: 10.1016/0169-4758(93)90135-3

Paillard, S., and Strauss, F. (1991). Analysis of the mechanism of interaction of simian Ku protein with DNA. Nucleic Acids Res. 19, 5619-5624. doi: 10.1093/ nar/19.20.5619

Petukhova, G., Stratton, S., and Sung, P. (1998). Catalysis of homologous DNA pairing by yeast Rad51 and Rad54 proteins. Nature 393, 91-94. doi: 10.1038/ 30037

Pezza, R. J., Voloshin, O. N., Vanevski, F., and Camerini-Otero, R. D. (2007). Hop2/Mnd1 acts on two critical steps in Dmc1-promoted homologous pairing. Genes Dev. 21, 1758-1766. doi: 10.1101/gad.1562907

Proudfoot, C., and McCulloch, R. (2006). Trypanosoma brucei DMC1 does not act in DNA recombination, repair or antigenic variation in bloodstream stage cells. Mol. Biochem. Parasitol. 145, 245-253. doi: 10.1016/j.molbiopara.2005. 10.007

Rahman, F., Tarique, M., and Tuteja, R. (2016). Plasmodium falciparum Bloom homologue, a nucleocytoplasmic protein, translocates in 3' to 5' direction and is essential for parasite growth. Biochim. Biophys. Acta 1864, 594-608. doi: 10.1016/j.bbapap.2016.02.016

Ratcliffe, H. L., and Geiman, Q. M. (1934). Amebiasis in reptiles. Science 79, 324-325. doi: 10.1126/science.79.2049.324

Regis-da-Silva, C. G., Freitas, J. M., Passos-Silva, D. G., Furtado, C., AugustoPinto, L., Pereira, M. T., et al. (2006). Characterization of the Trypanosoma cruzi Rad51 gene and its role in recombination events associated with the parasite resistance to ionizing radiation. Mol. Biochem. Parasitol. 149, 191-200. doi: 10.1016/j.molbiopara.2006.05.012

Roy, N., Bhattacharyya, S., Chakrabarty, S., Laskar, S., Babu, S. M., and Bhattacharyya, M. K. (2014). Dominant negative mutant of Plasmodium Rad51 causes reduced parasite burden in host by abrogating DNA double-strand break repair. Mol. Microbiol. 94, 353-366. doi: 10.1111/mmi.12762 
San Filippo, J., Sung, P., and Klein, H. (2008). Mechanism of eukaryotic homologous recombination. Annu. Rev. Biochem. 77, 229-257. doi: 10.1146/ annurev.biochem.77.061306.125255

Singh, N., Bhattacharya, A., and Bhattacharya, S. (2013). Homologous recombination occurs in Entamoeba and is enhanced during growth stress and stage conversion. PLOS ONE 8:e74465. doi: 10.1371/journal.pone.007 4465

Soulas-Sprauel, P., Rivera-Munoz, P., Malivert, L., Le Guyader, G., Abramowski, V., Revy, P., et al. (2007). V(D)J and immunoglobulin class switch recombinations: a paradigm to study the regulation of DNA end-joining. Oncogene 26, 7780-7791. doi: 10.1038/sj.onc. 1210875

Sung, P. (1997). Function of yeast Rad52 protein as a mediator between replication protein A and the Rad51 recombinase. J. Biol. Chem. 272, 28194-28197. doi: $10.1074 /$ jbc.272.45.28194

Takaku, M., Kainuma, T., Ishida-Takaku, T., Ishigami, S., Suzuki, H., Tashiro, S., et al. (2011). Halenaquinone, a chemical compound that specifically inhibits the secondary DNA binding of RAD51. Genes Cells 16, 427-436. doi: 10.1111/j. 1365-2443.2011.01494.x

Thorslund, T., Mcilwraith, M. J., Compton, S. A., Lekomtsev, S., Petronczki, M., Griffith, J. D., et al. (2010). The breast cancer tumor suppressor BRCA2 promotes the specific targeting of RAD51 to single-stranded DNA. Nat. Struct. Mol. Biol. 17, 1263-1265. doi: 10.1038/nsmb.1905

Ubeda, J. M., Legare, D., Raymond, F., Ouameur, A. A., Boisvert, S., Rigault, P., et al. (2008). Modulation of gene expression in drug resistant Leishmania is associated with gene amplification, gene deletion and chromosome aneuploidy. Genome Biol. 9:R115. doi: 10.1186/gb-2008-9-7-r115

Ubeda, J. M., Raymond, F., Mukherjee, A., Plourde, M., Gingras, H., Roy, G., et al. (2014). Genome-wide stochastic adaptive DNA amplification at direct and inverted DNA repeats in the parasite Leishmania. PLOS Biol. 12:e1001868. doi: 10.1371/journal.pbio.1001868

Vink, C., Rudenko, G., and Seifert, H. S. (2012). Microbial antigenic variation mediated by homologous DNA recombination. FEMS Microbiol. Rev. 36, 917-948. doi: 10.1111/j.1574-6976.2011.00321.x

Wang, H., and Xu, X. (2017). Microhomology-mediated end joining: new players join the team. Cell Biosci. 7:6. doi: 10.1186/s13578-017-0136-8

Yang, H., Jeffrey, P. D., Miller, J., Kinnucan, E., Sun, Y., Thoma, N. H., et al. (2002). BRCA2 function in DNA binding and recombination from a BRCA2-DSS1ssDNA structure. Science 297, 1837-1848. doi: 10.1126/science.297.5588.1837

Zhao, W., Saro, D., Hammel, M., Kwon, Y., Xu, Y., Rambo, R. P., et al. (2014). Mechanistic insights into the role of Hop2-Mnd1 in meiotic homologous DNA pairing. Nucleic Acids Res. 42, 906-917. doi: 10.1093/nar/gkt924

Zhao, W., Vaithiyalingam, S., San Filippo, J., Maranon, D. G., Jimenez-Sainz, J., Fontenay, G. V., et al. (2015). Promotion of BRCA2-dependent homologous recombination by DSS1 via RPA targeting and DNA mimicry. Mol. Cell. 59, 176-187. doi: 10.1016/j.molcel.2015.05.032

Conflict of Interest Statement: The authors declare that the research was conducted in the absence of any commercial or financial relationships that could be construed as a potential conflict of interest.

Copyright (c) 2017 Kelso, Waldvogel, Luthman and Sehorn. This is an open-access article distributed under the terms of the Creative Commons Attribution License (CC BY). The use, distribution or reproduction in other forums is permitted, provided the original author(s) or licensor are credited and that the original publication in this journal is cited, in accordance with accepted academic practice. No use, distribution or reproduction is permitted which does not comply with these terms. 